\title{
Ion Beam Microscopy: a Tool for Materials
}

\author{
L.C. Alves ${ }^{* * * *}$, V. Corregidor***, T. Pinheiro and L. Ferreira*** \\ *Campus Tecnológico e Nuclear, IST/ITN, Universidade Técnica de Lisboa E.N. 10, 2686-953 Sacavém \\ **Centro de Física Nuclear da Universidade de Lisboa, Av. Prof. Gama Pinto, n², 1949-003 Lisboa
}

Ion Beam Analytical techniques (IBA) using $\mathrm{MeV}$ charged particles are powerful techniques for the study of different type of samples in several science fields such as Material Science, microelectronics or biology/biomedicine due to its fine sensitivity, versatility and "non-destructive" characteristics. The possibility of beam focusing and beam scanning adds spatial resolution down to the $\mu \mathrm{m}$ level and imaging capabilities then allowing the IBA techniques to become microscopy techniques.

In the Nuclear Microprobe installed at IST/ITN several IBA techniques can be routinely used for materials characterization, the most common ones being PIXE (Particle Induced X-ray Emission), RBS (Rutherford Backscattering Spectrometry) and STIM (Scanning Transmission Ion Microscopy). Whether through their independent or combined use the most important thing of these techniques is the complementary information that they can grant. As any other X-ray spectroscopic technique $\mu$ PIXE can also provide elemental identification (for $\mathrm{Z}>12)$ but further present their spatial distribution in the sample as well as, for thin biological samples $(<20 \mu \mathrm{m})$, calculate their areal mass density. RBS on the other hand is able to probe sample in depth then allowing obtaining, for instance, elemental depth profile and at the same time sample matrix areal mass density. The combined use of PIXE and RBS then allows determining elemental concentration for thin biological samples. For the mentioned thin biological samples the ion beam energy loss when crossing them (base of the STIM technique), contains information on their density or thickness allowing unique information on its structure and morphology.

Advantages and draw backs can always be taken into account when comparing with similar or competitive techniques. This is the case of PIXE and SEM-EDS which is quite unfavorable for PIXE in the case of image spatial resolution, but quite favorable if elemental sensitivity is considered. Due to the much lower X-ray spectrum background, detection limits for PIXE reach the $\mu \mathrm{g} / \mathrm{g}$ level.

As an example of application, some of the results obtained for PE-g-HEMA films [1] are here shown. To allow their utilization as biomaterials for biomedical applications (e.g. drug delivery) apart from the needed mechanical properties and surface characteristics, biocompatibility of these materials is of fundamental importance. Regarding biocompatibility one important parameter to be assessed is its cytotoxicity that strongly depends on the contamination level at the surface. As shown in Fig. 1, MeV ion beam microscopy not only can provide major and trace element spatial distribution (combining PIXE and RBS data) but also valuable information on its near-surface structure (STIM). Furthermore, quantitative elemental analysis can be performed through the analysis of the PIXE spectra with sensitivity down to the $\mu \mathrm{g} / \mathrm{g}$ level as also revealed in Figure 1.

\section{References}

1. Ferreira L.M. et al., Rad. Phys. \& Chem., 81:1319-1323, 2012.

2. Alves L.C. et al., NIMB, 161:334-338, 2000. 

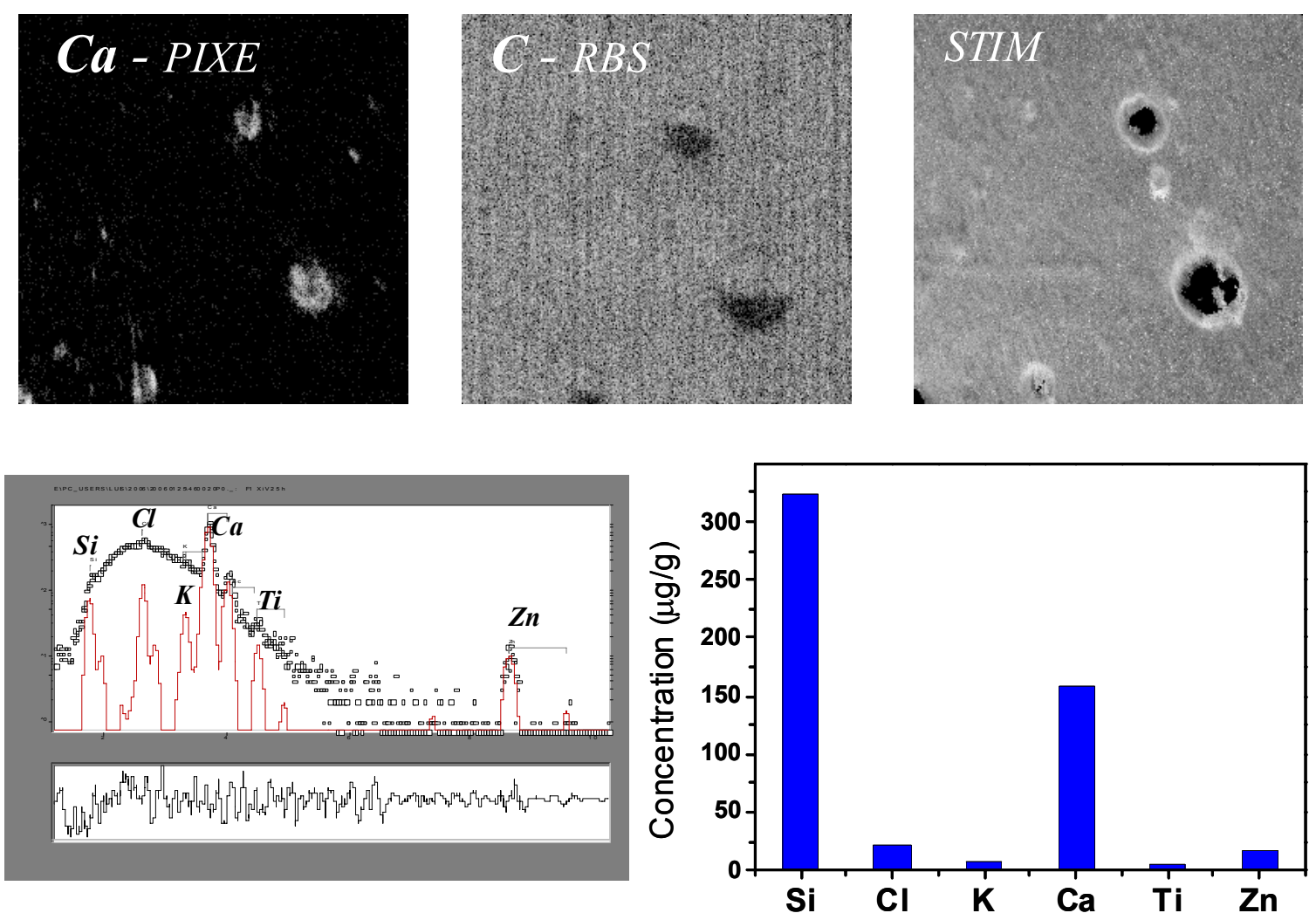

Figure 1 - Elemental distribution of $\mathrm{Ca}$ (obtained through PIXE), $\mathrm{C}$ (obtained through RBS) and surface structure of the PE-g-HEMA analysed film (obtained through STIM), during a 530x530 $\mu^{2}$ scanning of a $2 \mathrm{MeV}$ proton beam (top row). Also shown is the "average" PIXE spectra obtained during the beam scanning and the derived elemental concentrations (bottom row).

V. Corregidor acknowledges the funding support from the FCT-Ciência program. 